\title{
Graph Similarity based on Graph Fourier Distances
}

\author{
Eva Lagunas*, Antonio G. Marques ${ }^{\dagger}$, Symeon Chatzinotas*, Björn Ottersten* \\ *Interdisciplinary Centre for Security, Reliability and Trust (SnT), University of Luxembourg, Luxembourg \\ Email: \{eva.lagunas,symeon.chatzinotas,bjorn.ottersten\}@uni.lu ${ }^{\dagger}$ Department of Signal Theory and \\ Communications, King Juan Carlos University, Madrid, Spain \\ Email: antonio.garcia.marques@urjc.es
}

\begin{abstract}
Graph theory is a branch of mathematics which is gaining momentum in the signal processing community due to their ability to efficiently represent data defined on irregular domains. Quantifying the similarity between two different graphs is a crucial operation in many applications involving graphs, such as pattern recognition or social networks' analysis. This paper focuses on the graph similarity problem from the emerging graph Fourier domain, leveraging the spectral decomposition of the Laplacian matrices. In particular, we focus on the intuition that similar graphs should provide similar frequency representation for a particular graph signal. Similarly, we argue that the frequency responses of a particular graph filter applied to two similar graphs should be also similar. Supporting results based on numerical simulations support the aforementioned hypothesis and show that the proposed graph distances provide a new tool for comparing graphs in the frequency domain.
\end{abstract}

\section{INTRODUCTION}

Graphs are a convenient mathematical representation of networks. Relevant examples of networks with a strong impact on our daily lives include transportation systems (train lines or roads where graph edges represent streets and graph vertices represent road crossings), communications networks (telephone, computers, etc), social networks (LinkedIn, Facebook, etc) and biological networks (gene-to-gene, functional brain networks, etc), to name a few.

This paper focuses on the problem of comparing two different graphs and, in particular, on the definition of a new similarity metric to quantify the frequency domain similarity between two graphs. Having proper metrics to evaluate graph similarity is of high relevance in a number of applications, such as biological network comparison, web searching enhancement tools, and to match chemical structures. Focusing on conventional time-domain works, graph isomorphism has been widely investigated to claim that two graphs are identical, i.e. contain the same number of vertices which are connected in the same way [1]. While graph isomorphism provides a binary answer to the graph similarity, the emerging concept of graph similarity has been investigated for the last decade to provide a better assessment of the resemblance among graphs [2]. Generally speaking, the available literature on graph matching quantifies the difference between two graphs by the "cheapest" sequence of operations needed to transform one of the two graphs into the other (e.g. adding/deleting nodes or edges). However, the decision on the cost assigned to each operation is not trivial and has a deep impact on the final outcome. Alternative inexact graph matching techniques consider the use of a "graph distance", for instance [3], which collects all information related to a particular node in a signature vector and uses a node-assignment combined with a modified Euclidean distance to quantify the graph similarity. In addition, many of the state-of-the-art graph and sub-graph matching approaches are not robust to noisy and incomplete graphs. Despite the numerous works in the topic, a unifying and robust graph matching solution capable of comparing two graphs has not been yet provided.

The focus of this paper in on the case where the graphs to be compared have known node correspondence, i.e. they consist of the same number of nodes and those are aligned. One of the most relevant works in this area is reported in [4], where the pairwise node affinities are computed and compared. However, the proposed algorithm requires the computation of a node-by-node affinity, and the outcomes strongly depend on the method chosen to compute such affinity scores. In addition, the proposed metric to quantify the affinity scores requires matrix inversion and the definition of a constant to account for the influence between neighboring nodes.

In this paper we propose a novel graph similarity framework based on the spectral graph theory. In recent years, there has been significant contribution from the signal processing community towards the graph processing theory, by extrapolating spectral processing properties into the graph framework [5], [6]. Here, we particularly focus on the spectral decomposition of the graph Laplacian matrix which is known to bridge the discrete graph representation with the continuous spectral domain. In this context, we develop a novel graph Fourier based framework that allows us to obtain different methods for quantifying the similarity between two different graphs. In particular, we present a set of procedures which are evaluated and compared with [4] via numerical simulations.

The remainder of this paper is organized as follows. Section II introduces the graph notation and reviews the concept of graph Laplacian matrix (Section II-A) as well as the graph similarity metric proposed in [4] (Section II-B). After that, Section III presents the Laplacian matching framework and states the different proposed graph distances. Finally, support- 
ing numerical results are provided in Section IV, and Section $\mathrm{V}$ states the conclusion.

\section{PREliminaries: GraphS AND Similarity}

Let us consider a finite-size graph $\mathcal{G}=(\mathcal{N}, \mathcal{E}, \mathcal{W})$, consisting of a set of vertices $\mathcal{N}=\{1,2, \ldots, N\}$, a set of edges connecting the vertices $\mathcal{E} \subset V \times V$, such that $(n, m) \in \mathcal{E}$ if and only if vertex $n$ is connected to vertex $m$, and the corresponding edge weights $\mathcal{W} \subseteq N \times N$. If the graph $\mathcal{G}$ is undirected (which is the focus of this work), then the weight $w_{n m}$ connecting the edge $(n, m)$ is the same as the weight $w_{m n}$ connecting the edge $(m, n)$.

Any graph can be equivalently represented by its associated adjacency matrix $\mathbf{A} \in \mathbb{R}^{N \times N}$, whose entries are defined as

$$
A_{n m}= \begin{cases}w_{n m} & \text { if }(n, m) \in \mathcal{E} \\ 0 & \text { otherwise. }\end{cases}
$$

Clearly, the matrix $\mathbf{A}$ accounts for the structure of the graph in the sense that if $(n, m) \notin \mathcal{E}$, then $A_{n m}=0$.

\section{A. Graph Laplacian}

For undirected graphs, the elements of the Laplacian matrix $\mathbf{L} \in \mathbb{R}^{N \times N}$ associated with $\mathcal{G}$ are defined as

$$
L_{n m}= \begin{cases}\operatorname{deg}(n) & \text { if } n=m \\ -w_{n m} & \text { if }(n, m) \in \mathcal{E} \\ 0 & \text { otherwise, }\end{cases}
$$

where $\operatorname{deg}(n)$ stands for the degree of vertex $n$, which is defined as $\operatorname{deg}(n)=\sum w_{n m}$. Like the adjacency matrix $\mathbf{A}$, the Laplacian $\mathbf{L}$ is a sparse matrix that account for the structure of the graph. In fact, upon defining the degree matrix $\mathbf{D} \in \mathbb{R}^{N \times N}$ as a diagonal matrix which contains information about the degree of each vertex, the Laplacian matrix can be alternatively written as $\mathbf{L}=\mathbf{D}-\mathbf{A}$.

The Laplacian matrix is a symmetric, real-valued and sparse matrix. It can also be shown that the Laplacian is always positive semidefinite (PSD) -note that the definition in (2) guarantees $\mathbf{L}$ to be diagonal dominant-, and that the allones vector is an eigenvector whose associated eigenvalue is zero; i.e., by construction we have that $\mathbf{L} \mathbf{1}=\mathbf{0}$. In fact, the number eigenvalues of $\mathbf{L}$ that are zero (as well as the number of eigenvalues with very small magnitude) provide key information about the underlying structure of the graph, including the number of components (and connectivity) [7].

While the definition in (2), also referred to as combinatorial Laplacian, is the most widely used in the literature, alternative definitions for the Laplacian exist. These include the normalized Laplacian, the random-walk Laplacian, and the diagonally scaled Laplacian [7].

\section{B. State-of-the-Art: Delta-Connectivity}

D. Koutra et al. proposed in [4] the "Delta-Connectivity" metric to asses the similarity of two unweighted and aligned graphs (i.e., with known node correspondence). Essentially, the proposed algorithm first computes the pair-wise node affinities or influence of the two graphs and, in a second step, compares them using the root euclidean distance, also known as JeffriesMatusita distance. However, there exists many different ways to characterize the pair-wise node affinities and the decision of which one to use deeply affects the similarity result. In [4], the Belief Propagation algorithm is proposed, which computes the affinity matrix $\mathbf{S}$ as follows

$$
\mathbf{S}=\left[\mathbf{I}+\epsilon^{2} \mathbf{D}-\epsilon \mathbf{A}\right]^{-1},
$$

where $\mathbf{I}, \mathbf{A}$ and $\mathbf{D}$ are the identity, adjacency and degree matrices, respectively. The complexity of (3) is of significance, particularly for high-dimensional graphs, as it involves a matrix inversion. In addition, the algorithm requires the definition of the constant $\epsilon$, which captures the amount of influence between neighboring nodes to be considered.

\section{Proposed Graph Fourier Transform Metrics}

We seek a suitable similarity function for aligned graphs (weighted or unweighted) which effectively captures the spectral difference between aligned graphs with reduced complexity. Since a well-established theory on the spectral behavior of the graph Laplacian exits, we propose to focus on conventional distance metrics that can be applied to the spectral decomposition of the Laplacian matrices associated with the graphs.

Before moving into details, let us review the properties of a metric $\vartheta\left(\mathbf{A}_{1}, \mathbf{A}_{2}\right)$ to qualify as a distance:

- To be always non-negative: $\vartheta\left(\mathbf{A}_{1}, \mathbf{A}_{2}\right) \geq 0$.

- To be zero if and only if we are comparing the same graph: $\vartheta\left(\mathbf{A}_{1}, \mathbf{A}_{2}\right)=0$ if and only if $\mathbf{A}_{1}=\mathbf{A}_{2}$.

- To be symmetric: $\vartheta\left(\mathbf{A}_{1}, \mathbf{A}_{2}\right)=\vartheta\left(\mathbf{A}_{2}, \mathbf{A}_{1}\right)$.

- To satisfy the triangle inequality: $\vartheta\left(\mathbf{A}_{1}, \mathbf{A}_{3}\right) \leq$ $\vartheta\left(\mathbf{A}_{1}, \mathbf{A}_{2}\right)+\vartheta\left(\mathbf{A}_{2}, \mathbf{A}_{3}\right)$.

In the context of graph signal processing, given the eigendecomposition of the Laplacian matrix

$$
\mathbf{L}=\mathbf{V} \operatorname{diag}\left(\lambda_{1}, \ldots, \lambda_{N}\right) \mathbf{V}^{T}
$$

a concept of particular relevance is that of the Graph Fourier Transform (GFT). As in classical signal processing, the GFT is used to provided an alternative representation for graph signals and graph filters and, equally important, transforms filtering in the graph domain into multiplication in the frequency domain; see, e.g, [8]. A noticeable difference relative to classical signal processing is that the GFT for signals is not the same than the GFT for filters [9]. In particular, given a signal $\mathbf{x} \in \mathbb{R}^{N}$ the graph Fourier transform that maps $\mathbf{x}$ to the $N$-dimensional frequency domain is given by the square orthonormal matrix V. Differently, given a vector of filter coefficients $\mathbf{h} \in \mathbb{R}^{N}$, the graph Fourier transform that maps $\mathbf{h}$ to the $N$-dimensional frequency domain is given by the square Vandermonde matrix $\boldsymbol{\Psi}$, where $[\boldsymbol{\Psi}]_{i, j}=\lambda_{i}^{j-1}$ [9]. 
Our contribution is then to use these two GFT matrices to propose new graph similarity matrices. In particular, consider two graphs $\mathcal{G}_{1}$ and $\mathcal{G}_{2}$ described by $\mathbf{L}_{1}$ and $\mathbf{L}_{2}$, respectively. Associated with each of the Laplacians, we can define $\left(\mathbf{V}_{1}, \mathbf{\Psi}_{1}\right)$ as well as $\left(\mathbf{V}_{2}, \mathbf{\Psi}_{2}\right)$. Using those, we propose the following two distances

$$
\begin{aligned}
& d^{G F T-S}\left(\mathcal{G}_{1}, \mathcal{G}_{2}\right)=\vartheta\left(\mathbf{V}_{1}, \mathbf{V}_{2}\right) \\
& d^{G F T-F}\left(\mathcal{G}_{1}, \mathcal{G}_{2}\right)=\vartheta\left(\mathbf{\Psi}_{1}, \boldsymbol{\Psi}_{2}\right) .
\end{aligned}
$$

Intuitively, if two graphs $\mathcal{G}_{1}$ and $\mathcal{G}_{2}$ are close when using the distance in (5), it means that the frequency representation of signals defined on those graphs is similar. From a different perspective, having $d^{G F T-S}\left(\mathcal{G}_{1}, \mathcal{G}_{2}\right)$ small means that matrices $\mathbf{L}_{1}$ and $\mathbf{L}_{2}$ are close to be simultaneously diagonalizable, and, therefore, that the Laplacian $\mathbf{L}_{1}$ can be well approximated as a matrix polynomial of $\mathbf{L}_{2}$, and viceversa (see [9] for a related problem).

On the other hand, if two graphs $\mathcal{G}_{1}$ and $\mathcal{G}_{2}$ are close under the distance in (6), it means that the frequency representation of filter taps associated with a filter defined either on $\mathbf{L}_{1}$ or $\mathbf{L}_{2}$ are similar. In other words, if one designs the coefficients of a graph filter to obtain a desired frequency response (say low-pass) on $\mathbf{L}_{1}$, the same filter coefficients will also give rise to a low-pass response on $\mathbf{L}_{2}$ provided that $d^{G F T-F}\left(\mathcal{G}_{1}, \mathcal{G}_{2}\right)$ is small.

For the function $\vartheta(.,$.$) , we consider the following distances:$

- Frobenius distance: Sometimes also called the Euclidean norm for matrices, is the extension of the usual distance applied in the vector space to the matrix space. The Frobenius distance is defined by,

$$
\begin{aligned}
d_{\text {fro }}\left(\mathbf{A}_{1}, \mathbf{A}_{2}\right)= & \left\|\mathbf{A}_{1}-\mathbf{A}_{2}\right\|_{F}= \\
& \sqrt{\sum_{n=1}^{N} \sum_{m=1}^{N}\left|\left[\mathbf{A}_{1}\right]_{n, m}-\left[\mathbf{A}_{2}\right]_{n, m}\right|^{2}} .
\end{aligned}
$$

This is the simplest and fastest to compute distance, involving a complexity $O\left(N^{2}\right)$ resulting from simple point-wise multiplications and addition of matrix entries. This complexity can be further reduced by exploiting the inherent sparsity in $\mathbf{A}_{1}$ and $\mathbf{A}_{2}$. However, the Frobenius distance treats the matrices simply as vectors and, thus, completely disregards other properties that $\mathbf{A}_{1}$ and $\mathbf{A}_{2}$ may satisfy, such as the inherent semi-definite property of the Laplacian matrices.

- Jeffries-Matusita distance: Similar to the Frobenius distance, but it generally provides better results since it introduces the square root of the matrix elements, which makes the distance more sensitive to small differences.
The Jeffries-Matusita distance is defined as follows,

$$
\begin{aligned}
& d_{\mathrm{JM}}\left(\mathbf{A}_{1}, \mathbf{A}_{2}\right)=\left\|\mathbf{A}_{1}-\mathbf{A}_{2}\right\|_{J M}= \\
& \sqrt{\sum_{n=1}^{N} \sum_{m=1}^{N} \mid \sqrt{\left[\mathbf{A}_{1}\right]_{n, m}}-\sqrt{\left.\left[\mathbf{A}_{2}\right]_{n, m}\right|^{2}}} .
\end{aligned}
$$

The computational complexity of the Jeffries-Matusita distance is yet driven by the square operation, i.e. $O\left(N^{2}\right)$.

One can also consider computing the similarity metric directly to the Laplacian matrices, which are known to account for the local structure of the graph. This is,

$$
d^{G F T-L}\left(\mathcal{G}_{1}, \mathcal{G}_{2}\right)=\vartheta\left(\mathbf{L}_{1}, \mathbf{L}_{2}\right)
$$

Given the positive-semidefinite characteristics of Laplacian matrices, we suggest to use in this case the geodesic distance, which matches better curved surfaces instead of plannar measurements. Note that the manifold of positive-semidefinite matrices is a convex cone structure and thus, plannar distances like the Frobenius norm fail in capturing the true distances. The Geodesic distance [10] is defined as,

$$
d_{\text {geo }}\left(\mathbf{A}_{1}, \mathbf{A}_{2}\right)=\sqrt{\sum_{1 \leq i \leq N}\left|\log \lambda_{i}\right|^{2}},
$$

where $\lambda_{1}, \lambda_{2}, \ldots, \lambda_{N}$ are the generalized eigenvalues of the two matrices, i.e.,

$$
\mathbf{A}_{1} \boldsymbol{v}_{n}=\lambda_{n} \mathbf{A}_{2} \boldsymbol{v}_{n}, \quad n=1, \ldots, N,
$$

with $\boldsymbol{v}_{1}, \boldsymbol{v}_{2}, \ldots, \boldsymbol{v}_{N}$ being the generalized eigenvectors. The computational complexity of (10) accounts to $O\left(N^{3}\right)$ as it involves a Generalized Eigenvalue Decomposition (GED).

However, the Laplacian matrix associated with sparsely connected graphs tends to have several eigenvalues of very small magnitude. The computation of the eigenvectors is thus very sensitive to matrix singularity and, as a consequence, cannot be directly applied to Laplacian matrices.

To bypass the the ill-conditioned behavior of the Laplacian matrices, we propose the to use a "Regularized Laplacian", which considers a regularized factor, $\alpha>0$ to be added to each of the Laplacian matrices under consideration,

$$
\begin{aligned}
\hat{\mathbf{L}}_{1} & =\mathbf{L}_{1}+\alpha \mathbf{I}_{N \times N} \\
\hat{\mathbf{L}}_{2} & =\mathbf{L}_{2}+\alpha \mathbf{I}_{N \times N} .
\end{aligned}
$$

Clearly, the eigenvalues of the matrix $\hat{\mathbf{L}}_{1}$ are those of the matrix $\mathbf{L}_{1}$ shifted by the positive constant $\alpha$, so that all the eigenvalues of $\hat{\mathbf{L}}_{1}$ (as well as those of $\hat{\mathbf{L}}_{2}$ ) are greater than zero and increasing with $\alpha$.

Remark: Alternative generalizations and DeltaConnectivity. The definitions in (12) and (13) coincide with one the forms of the generalized Laplacians. Alternatively, one could consider the generalization

$$
\tilde{\mathbf{L}}_{1}=\mathbf{L}_{1}+\alpha \mathbf{D}_{1}
$$




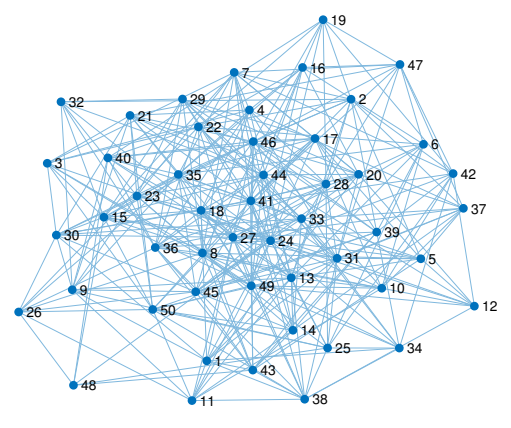

Fig. 2: Example of graph obtained with Erdos-Renyi model with $N=50$

$$
\tilde{\mathbf{L}}_{2}=\mathbf{L}_{2}+\alpha \mathbf{D}_{2}
$$

Since $\tilde{\mathbf{L}}_{1}$ and $\tilde{\mathbf{L}}_{2}$ are symmetric and strictly diagonal dominant, all the eigenvalues of are greater than zero and increasing with $\alpha$. Interestingly, the definition in (14) can be related to that in (3). Specifically, upon setting $\alpha=1-\epsilon$, one has that

$$
\mathbf{S}=\left(\mathbf{I}+\epsilon \tilde{\mathbf{L}}_{1}\right)^{-1} .
$$

Since in the context of graph signal processing, graph filters are defined as polynomials of the so-called graph shift operator, a sparse matrix accounting for the structure of the graph [8], [9], the above equation reveals that the affinity matrix S, which serves as basis of the "Delta-Connectivity" similarity, can be understood as a linear graph filter on the shift operator $\tilde{\mathbf{L}}_{1}$. Hence, by choosing different types of shift operators (generalized Laplacians) as well as different types of filters (low vs. high pass, single-pole vs. single-zero) one can encode a span of notions of similarities among graphs. This issue will be subject of future research.

\section{Simulation Results}

To gain some intuition on the proposed metrics, preliminary simulations are run. To that end, we have used the Erdos-Renyi model to generate a set of 8 random graphs with $N=50$ nodes and different edge set $\mathcal{E}$. An example of such graphs is depicted in Fig. 2.

Fig. 1 shows the distance results obtained with the proposed graph Fourier distances with Frobenius distance and JeffriesMatusita distance, and the results are compared with the geodesic distance computed over the Laplacian matrices (Fig. 1(c) and the Delta-Connectivity algorithm [4] (Fig. 1(f)). First of all, from Fig. 1 it can be observed that the three proposed metrics based on the Laplacian matching behave well, providing symmetric distance matrix with zero-diagonal elements. Secondly, Fig. 1 shows how the proposed spectralbased graph metrics capture the graph similarity achieving results which are comparable to the state-of-the-art without the need of computing the matrix of pairwise node affinity scores which is complex, time consuming and subject to the definition of $\epsilon$. Although a method to speed-up the affinity computation is proposed in [4], still the resulting matrix needs to be compared
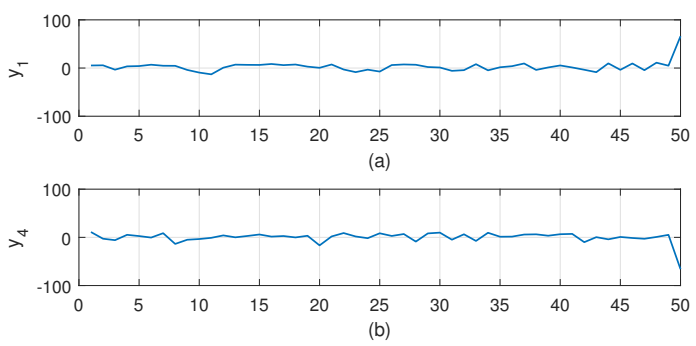

Fig. 3: Frequency representation of graph signal on non-similar graphs: (a) $\mathcal{G}_{1}$ and (b) $\mathcal{G}_{4}$
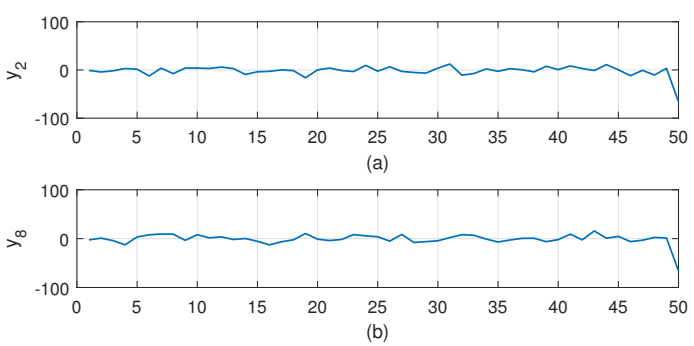

Fig. 4: Frequency representation of graph signal on similar graphs: (a) $\mathcal{G}_{2}$ and (b) $\mathcal{G}_{8}$

with a particular distance. The methods proposed in this paper are single-step metrics applied directly at the GFT of the Laplacian matrices.

Next, we take the pair of graphs $\mathcal{G}_{1}$ and $\mathcal{G}_{4}$ from the previous set, which seem to have distant graph Fourier transforms, $\mathbf{V}_{1}$ and $\mathbf{V}_{4}$, according to the previous results. We assume a certain graph signal $\mathbf{x} \in \mathbb{R}^{N}$ which is randomly generated with values uniformly distributed between $[0,20]$ and we compute the GFTs as $\mathbf{y}_{1}=\mathbf{V}_{1}^{-1} \mathbf{x}$ and $\mathbf{y}_{4}=\mathbf{V}_{4}^{-1} \mathbf{x}$. The resulting frequency domain responses are depicted in Fig. 3. It can be observed that the non-similarity in Vs translates into nonsimilar Fourier representations. Similarly, we have taken the pair of graphs $\mathcal{G}_{2}$ and $\mathcal{G}_{8}$ from the previous set, which have very similar $\mathbf{V}_{2}$ and $\mathbf{V}_{8}$, according to the previous results. We compute the GFTs as $\mathbf{y}_{2}=\mathbf{V}_{2}^{-1} \mathbf{x}$ and $\mathbf{y}_{8}=\mathbf{V}_{8}^{-1} \mathbf{x}$ using the same graph signal $\mathbf{x}$ and we show the obtained results in Fig. 4. Clearly, the obtained frequency responses are very similar, thus confirming our hypothesis.

Fig. 5 illustrates the frequency response of a filter composed of 5 taps, all equal to 1 , applied on graphs $\mathcal{G}_{5}$ and $\mathcal{G}_{7}$, which according to previous results have shown strong similarities in $d^{G F T-F}$. It can be observed that the filters' spectrum looks almost identical thus proving the benefits of the proposed GFT distances.

\section{CONCLUSION}

This paper proposed a new framework for assessing the similarity between aligned graphs (i.e. with node correspondence) based on the concept of graph spectral transfromation of the Laplacian matrix. The motivation for this work is to 


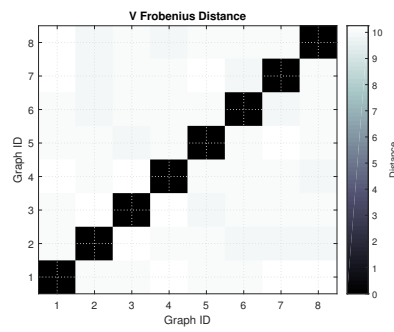

(a) $d^{G F T-S}$ with Frobenius

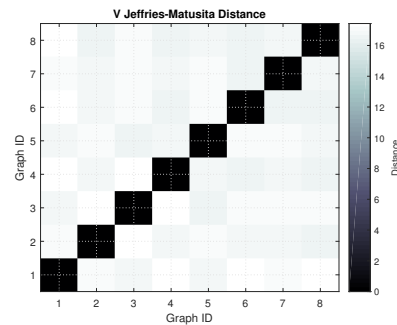

(d) $d^{G F T-S}$ with Jeffries-Matusita

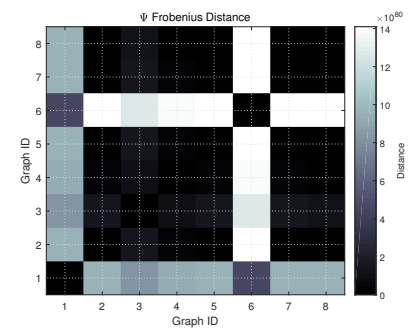

(b) $d^{G F T-F}$ with Frobenius

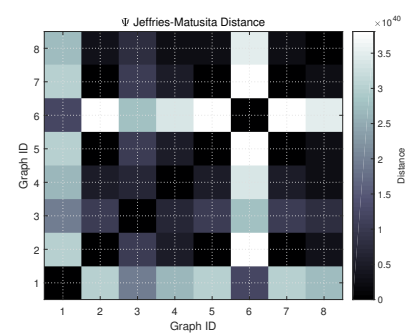

(e) $d^{G F T-F}$ with Jeffries-Matusita

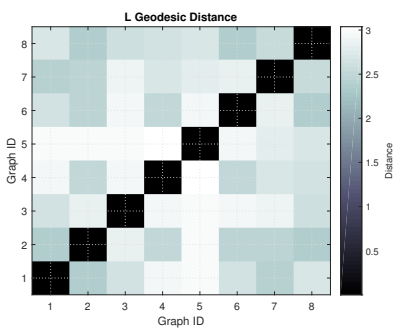

(c) $d^{G F T-L}$ with Geodesic

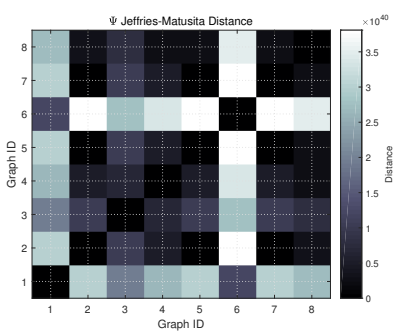

(f) Delta-Connectivity [4]

Fig. 1: Comparison of different distances.

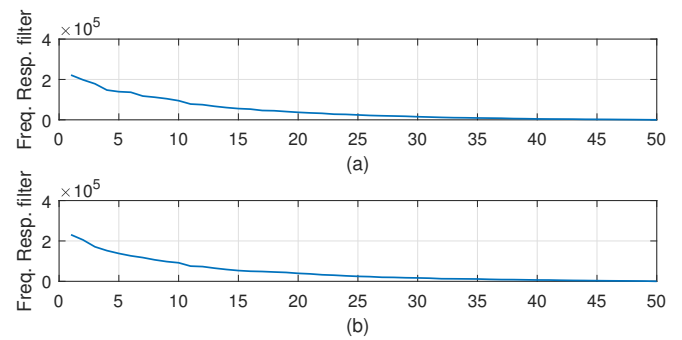

Fig. 5: Frequency representation of graph filter on similar graphs: (a) $\mathcal{G}_{5}$ and (b) $\mathcal{G}_{7}$

exploit current findings in spectral graph theory to improve the available graph similarity tools. Different distances have been proposed and preliminary simulations assessing their performance have been presented. When comparing the proposed metrics with the most relevant state-of-the-art technique, the schemes presented in the paper show a good balance between complexity and performance. Future work includes consideration of more general matrix polynomial distances, analytical characterization of the distance for particular types of random graph models, and extensions of the definitions for graphs where node correspondences are not known.

\section{ACKNOWLEDGMENT}

A.G. Marques was supported by the Spanish MINECO grants TEC2013-41604-R and TEC2016-75361-R. Other authors received funding from the European Research Council
(ERC) under the EU H2020 research and innovation programme (grant agreement No. 742648).

\section{REFERENCES}

[1] L. Chen, "Graph Isomorphism and Identification Matrices: Parallel Algorithms," IEEE Transactions on Parallel and Distributed Systems, vol. 7, no. 3, pp. 308-319, 1996.

[2] D. Conte, P. Foggia, C. Sansone, and M. Vento, "Thirty Years of Graph Matching in Pattern Recognition," International Journal of Pattern Recognition and Artificial Intelligence, vol. 18, no. 3, pp. 265-298, 2004.

[3] S. Jouili and S. Tabbone, "Graph Matching Based on Node Signatures," International Workshop on Graphics Recognition (GREC), La Rochelle, France, pp. 154-163, Jul. 2009.

[4] D. Koutra, N. Shah, J.T Vogelstein, B. Gallagher, and C. Faloutsos, "A Principled Massive-Graph Similarity Function with Attribution," ACM Transactions on Knowledge Discovery from Data (TKDD), vol. 10, no. 3, pp. 1-43, Feb. 2016.

[5] D.I. Shuman, S.K. Narang, P. Frossard, A. Ortega, and P. Vandergheynst, "The Emerging Field of Signal Processing on Graphs: Extending HighDimensional Data Analysis to Networks and Other Irregular Domains," IEEE Signal Process. Mag., vol. 130, no. 3, pp. 83-98, 2013.

[6] A.G. Marques, S. Segarra, G. Leus, and A. Ribeiro, "Stationary Graph Processes and Spectral Estimation," IEEE Transactions on Signal Processing, vol. 65, no. 22, pp. 5911-5926, Nov. 2017.

[7] P. Djuric and C. Richard, Cooperative and Graph Signal Processing, Academic Press Inc., New York and London, 2018.

[8] A. Sandryhaila and J.M.F. Moura, "Discrete signal processing on graphs," IEEE Transactions on Signal Processing, vol. 61, no. 7, pp. 1644-1656, Apr. 2013

[9] S. Segarra, A. G. Marques, and A. Ribeiro, "Optimal graph-filter design and applications to distributed linear network operators," IEEE Transactions on Signal Processing, vol. 65, no. 15, pp. 4117-4131, Aug. 2017.

[10] S. Helgason, Differential Geometry and Symmetric Spaces, Academic Press Inc., New York and London, 1962. 